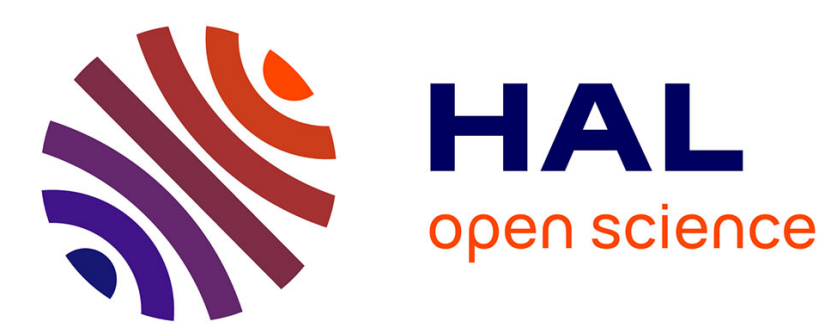

\title{
Les déplacés du cyclone Katrina: de l'exode à la migration
}

Francois Mancebo

\section{To cite this version:}

Francois Mancebo. Les déplacés du cyclone Katrina: de l'exode à la migration. Espaces et sociétés (Paris, France), 2009, 139 (4), pp.141. 10.3917/esp.139.0141 . hal-02017703

\section{HAL Id: hal-02017703 \\ https://hal.univ-reims.fr/hal-02017703}

Submitted on 14 Feb 2019

HAL is a multi-disciplinary open access archive for the deposit and dissemination of scientific research documents, whether they are published or not. The documents may come from teaching and research institutions in France or abroad, or from public or private research centers.
L'archive ouverte pluridisciplinaire HAL, est destinée au dépôt et à la diffusion de documents scientifiques de niveau recherche, publiés ou non, émanant des établissements d'enseignement et de recherche français ou étrangers, des laboratoires publics ou privés. 


\title{
LES DÉPLACÉS DU CYCLONE KATRINA : DE L'EXODE À LA MIGRATION \\ François Mancebo
}

\author{
ERES | « Espaces et sociétés »
}

2009/4 n 139 | pages 141 à 158

ISSN 0014-0481

ISBN 9782749211602

Article disponible en ligne à l'adresse :

https://www.cairn.info/revue-espaces-et-societes-2009-4-page-141.htm

\section{Pour citer cet article :}

François Mancebo, «Les déplacés du cyclone Katrina : de l'exode à la migration 》, Espaces et sociétés 2009/4 ( $\left.\mathrm{n}^{\circ} 139\right)$, p. 141-158.

DOI 10.3917/esp.139.0141

Distribution électronique Cairn.info pour ERES.

(C) ERES. Tous droits réservés pour tous pays.

La reproduction ou représentation de cet article, notamment par photocopie, n'est autorisée que dans les limites des conditions générales d'utilisation du site ou, le cas échéant, des conditions générales de la licence souscrite par votre établissement. Toute autre reproduction ou représentation, en tout ou partie, sous quelque forme et de quelque manière que ce soit, est interdite sauf accord préalable et écrit de l'éditeur, en dehors des cas prévus par la législation en vigueur en France. Il est précisé que son stockage dans une base de données est également interdit. 
II

HORS DOSSIER 


\title{
DOA \\ Les déplacés du cyclone Katrina : de l'exode à la migration
}

François Mancebo

\begin{abstract}
Dans le cas du cyclone Katrina, une catastrophe environnementale a été à l'origine d'un des plus vastes mouvements de population aux États-Unis. Catastrophe environnementale et non catastrophe « naturelle », car s'il existe des phénomènes naturels dont les cyclones font partie, leurs effets catastrophiques, dépendent de la manière dont les hommes aménagent leur espace de vie et du risque qu'ils acceptent de prendre : choix historique de fonder la ville là plutôt qu'ailleurs, choix individuels cumulatifs d'y habiter, choix sociétaux et choix institutionnels dans le mode d'urbanisation.

Une catastrophe «naturelle » est donc, fondamentalement, une catastrophe «humaine » en ce qu'elle résulte de choix d'exposition ou non à l'aléa. Katrina montre que le risque n'est pas une menace extérieure, mais bien cet élément constitutif de la société mis en évidence par Ulrich Beck (Beck, 2003). Ainsi, les marécages et les mangroves de Louisiane, qui jouent
\end{abstract}

François Mancebo, professeur (aménagement, urbanisme, développement durable), université Grenoble 1 Joseph Fourier, IGA/UMR Pacte, CNRS.

francois.mancebo@ujf-grenoble.fr 
un rôle de protection contre les cyclones en absorbant une grande partie de l'énergie cinétique des cyclones à la manière dont une pelouse épaisse absorbe la puissance d'un jet d'eau, se sont beaucoup réduits sous l'action humaine, depuis les années trente. Ainsi, également, les deux énormes canaux que sont le MRGO et l'Industrial Canal ont été orientés de telle manière qu'ils canalisent et concentrent la puissance des cyclones selon le principe d'une voie d'accélération, percutant de plein fouet en fin de course les levées au cœur de la ville. Enfin, La Nouvelle-Orléans, située en dessous du niveau de la mer, a naturellement tendance à garder les eaux qui l'envahissent sans cesse. Des pompes énormes expulsaient ces eaux en permanence dans le lac Pontchartrain. Or, celles-ci, ont été placées à des endroits vulnérables : elles ont cessé de fonctionner dès que les premières levées ont cédé. C'est ainsi que La Nouvelle-Orléans a été inondée sous l'action combinée d'un phénomène naturel extrême et de politiques d'aménagement aberrantes.

À l'issue du passage du cyclone, près de $80 \%$ de la population est partie ou a été évacuée (McQuaid, 2006). En janvier 2006, la Federal Emergency Management Agency (FEMA) évaluait le nombre de déplacés à plus de 2 millions. Fin 2006, plus de $40 \%$ n'étaient pas revenus ; $60 \%$ pour l'aire métropolitaine de La Nouvelle-Orléans. Que sont-ils devenus?

De nombreux travaux ont été menés pour comprendre les causes de cette catastrophe sur le plan de l'aménagement, du régime d'urbanisation ou encore des logiques d'acteurs sous-jacentes (Mancebo, 2006). D'autres ont interrogé les dynamiques de la reconstruction dans une perspective géographique (Cadoul et Maret, 2008). Mais peu a été écrit sur ce que sont devenues les personnes déplacées ou sur les forces en jeu dans ce déplacement massif de population. Or, il semble difficile de comprendre ce qui s'est passé en août 2005 à La Nouvelle-Orléans ou d'imaginer le devenir de cette ville, sans prendre en compte ceux qui ne sont pas revenus. Cet article ébauche ce travail à partir de documents et de données multiples venant de différents horizons (administratifs, politiques, économiques), de statistiques, d'enquêtes de terrain.

\section{Internally Displaced People}

Il convient d'examiner brièvement comment qualifier l'exil des déplacés. En 1985, un rapport du PNUED (Progamme des Nations unies pour l'Environnement et le Développement) a établi un lien direct entre mouvements de populations et catastrophe environnementale, dans le contexte de la grande sécheresse du Sahel (El-Hinnawi, 1985) : «[...] Les réfugiés environnementaux sont des personnes qui ont été obligées de quitter leur habitat traditionnel, temporairement ou de manière permanente, à cause d'une catastrophe environnementale qui a mis en danger leur existence ou qui a affecté 
notablement leurs conditions de vie ${ }^{1}$. En ce sens, les victimes du cyclone Katrina pourraient être qualifiées de réfugiés environnementaux.

Mais, dans le cas de Katrina, l'usage du terme «réfugié » a vite été contesté par les dirigeants afro-américains des droits civiques : «Ce ne sont pas des réfugiés ; ce sont des citoyens de Louisiane et du Mississippi, payant leurs impôts, et non des réfugiés errants en quête de charité » ${ }^{2}$ (Broder, 2005). De manière plus générale, nombre d'auteurs contestent l'usage même du terme réfugié environnemental, au motif qu'il crée une confusion avec les réfugiés politiques, fragilisant le statut de ces derniers (McGregor, 1995).

Dans cette perspective, certains suggèrent d'utiliser le terme de migrants environnementaux, lorsque les déplacements de populations sont dus initialement à une dégradation du milieu de vie (Swain, 1996). Dans le cas présent, on a affaire à une population très majoritairement pauvre, qui l'était déjà avant le passage du cyclone Katrina. Pourtant, malgré des conditions de vie extrêmement difficiles, elle persistait à vivre à La Nouvelle-Orléans. C'est bien la catastrophe qui est à l'origine de leur départ. Ce qui correspond plus au réfugié environnemental, qui fait partie de "populations ou de groupes sociaux déplacés par contrainte, sous l'effet de bouleversements environnementaux soudains et irréversibles » (Suhrke et Visentin, 1991).

Si l'on considère les personnes déplacées comme des migrants, alors elles n'ont droit à aucun statut, ni protection spécifique, alors que leur dénuement est aussi réel que celui des réfugiés. Avec ironie, Simms and Conisbee nomment legal gypsies de tels «migrants» (Conisbee et Simms, 2003).

En réalité, il existe un statut et des principes pour protéger les droits de personnes telles que les victimes de Katrina: l'United Nations Guiding Principles on Internal Displacement, fondé sur 30 principes directeurs prenant en compte le départ, les conditions de vie des personnes déplacées, puis le retour ou la migration définitive. Il nomme les populations concernées d'un terme spécifique : «internally-displaced people » (littéralement personnes déplacées à l'intérieur du pays), figuré la plupart du temps par l'abréviation IDPs. Cette catégorie correspond parfaitement aux victimes de Katrina. C'est elle que nous utiliserons dans cet article [http://www.ushrnet work.org/files/ushrn/images/linkfiles/HOLD_THE_US_ACCOUN TABLE_Membership_Petition_Final\%20Final.pdf].

1. «[...] Environmental refugees are defined as those people who have been forced to leave their traditional habitat, temporarily or permanently, because of a marked environmental disruption that jeopardized their existence and/or seriously affected the quality of their life."

2. "These are not refugees; they are citizens of Louisiana and Mississippi, tax-paying citizens. They are not refugees wandering somewhere looking for charity. They are victims of neglect and a situation they should have never been put in in the first place. » 
Si le gouvernement américain ne reconnaît pas formellement aux victimes de Katrina le statut d'IDPs, le dispositif mis en place, pour leur venir en aide les désigne implicitement comme tels. C'est le Stafford Disaster Relief and Emergency Assistance Act qui fournit, depuis 1988, le cadre légal de la réponse fédérale dans les catastrophes majeures. Il autorise le président à déclarer un état de catastrophe naturelle qui autorise les agences fédérales concernées - principalement la Federal Emergency Management Agency (FEMA) - à assister les États affectés et leurs gouvernements locaux. Bien que le Stafford Act ne prend pas explicitement en charge le long terme, le National Response Plan - document officiel qui définit les responsabilités des différentes agences gouvernementales - inclut une annexe intitulée LongTerm Community Recovery and Mitigation (restauration et soulagement à long terme des communautés locales) dont les principes évoquent fortement les IDPs.

\section{Peu de retours, une redistribution générale}

La plupart des IDPs de Katrina ont été accueillis à l'intérieur de la Louisiane ou dans les États voisins. Le Texas est l'État qui en a reçu la grande majorité avec plus de 250000 personnes, mais l'Arkansas en a également reçu plus de 60000 . D'autres sont partis beaucoup plus loin, se répartissant dans 30 États (White House, 2006) dont l'Alabama (25 000), le Tennessee (15 000), la Géorgie (15 000), l'Oklahoma (5 000), le New Jersey (3 000), le Colorado (2 000), la Floride (1 000). Ces départs massifs ont eu un impact considérable sur la démographie de La Nouvelle-Orléans. La ville, auparavant en forte croissance démographique avec plus de 470000 habitants, s'est réduite considérablement. En août 2006, avec moins de 200000 habitants, $60 \%$ des anciens résidents de La Nouvelle-Orléans manquaient (The Brookings Institution and Greater New Orleans Community Data Center, 2007).

Une estimation de 2006 montrait que $60 \%$ des IDPs continuaient à vivre dans les trois États du Texas, du Mississippi et de la Géorgie, et $25 \%$ en Floride, Alabama, Tennessee, Californie, Caroline du Nord, Illinois, Arkansas et Virginie (Louisiana Family Recovery Corps, 2007). Ces chiffres signifient deux choses : beaucoup d'IDPs ne sont pas rentrés, se transformant de fait en migrants environnementaux ; leur distribution géographique plus d'un an après la catastrophe n'est pas la même que la distribution initiale. Cela signifie que nombre d'IDPs sont partis s'installer définitivement dans d'autres États, sans rentrer à La Nouvelle-Orléans ni rester à l'endroit où ils ont été déplacés. Quelles sont les raisons de ce phénomène ? 


\section{L'IMPOSSIBLE RETOUR}

En 2006, une étude menée par le bureau du recensement américain, montre que $40 \%$ seulement des personnes qui ne sont pas rentrées désirent retourner dans leur ancien lieu de vie (us Census Bureau, 2006). La majorité d'entre elles est pauvre ou vulnérable. Celles qui envisagent de vivre ailleurs - la grande majorité des IDPs - sont plus autonomes, plus riches ou plus jeunes. On pourrait s'attendre à ce que leur décision soit motivée par les opportunités (bon travail, meilleures conditions de vie, etc.) offertes par leur nouveau milieu de vie. Il n'en est rien. Leur décision est généralement fondée sur des barrières et des contraintes, les empêchant de revenir. Parmi les raisons le plus souvent évoquées figurent le manque d'argent pour le déménagement et l'installation, les difficultés à trouver un logement sur place, les doutes sur l'efficacité du nouveau système de levées.

\section{Où se loger? Entre prix inabordables et gentrification de la ville}

Le passage de Katrina a détruit 300000 logements à La NouvelleOrléans. Mais la FEMA a aussi classé 60000 immeubles et maisons en état de péril, c'est-à-dire inhabitables et destinés à être démolis (Lipton, 2005). Avant l'ouragan, il existait une grande hétérogénéité dans le type d'urbanisation et le profil socio-culturel des habitants entre les quartiers de La Nouvelle-Orléans. Après Katrina, cette hétérogénéité s'est révélée dans les différences de vulnérabilité des bâtis et dans les inégalités spatiales de la reconstruction. Les plus anciens quartiers - French Quarter ou Garden District - sont situés sur les sites les plus élevés. Ce sont des espaces touristiques habités par des populations aisées. Fort bien entretenus, ils n'ont que peu souffert. Il existe aussi des quartiers résidentiels dans des espaces très soumis aux inondations, soit parce qu'ils sont très bas par rapport au niveau de la mer, tels Holy Cross, Esplanade Ridge, Broadmoor ou Jackson Barrack, soit parce qu'ils sont à proximité immédiate du Lac Pontchartrain tels Jefferson Parish, Lakeview, Gentilly ou New Orleans East. Ils ont été submergés sous près de 2,50 mètres d'eau. Mais les habitants de ces lieux avaient la capacité financière de reconstruire, ce qu'ils ont fait malgré la dangerosité du site. Contrairement à un cliché tenace, les quartiers les plus pauvres formés de maisonnettes en bois ne sont pas ceux qui ont été les plus touchés par les inondations. Par contre, l'habitat précaire qui s'y trouvait n'a résisté ni au vent ni à la montée des eaux. En ces endroits, peu de réhabilitations ont été réalisées car les habitants n'en ont pas les moyens, alors qu'elles seraient relativement peu coûteuses au regard d'autres quartiers. C'est ainsi que le Lower 9th ward, l'un des quartiers les plus déshérités, ressemble toujours à une ville morte. De surcroît, les fonds privés, mais aussi publics se sont orientés majoritairement vers les sites les plus rentables ou présentant un intérêt économique. 
De fait, les IDPs qui essaient de revenir ont d'énormes difficultés pour trouver un logement abordable. À La Nouvelle-Orléans, les loyers ont augmenté de $40 \%$ à $200 \%$ une année après le cyclone (Meitrodt, 2006). Il en résulte une difficulté pour ceux qui désirent revenir à trouver des logements disponibles. Il semble y avoir, dans ce domaine, une instrumentalisation de la catastrophe par les autorités locales, d'État et fédérales dans une stratégie concertée d'exclusion des populations les plus dérangeantes. La FEMA a ainsi admis que leur évaluation du nombre de logements en état de péril était largement excessive, car la plupart des inspections n'étaient que des rapid exterior inspections (Lipton, op. cit.). Or, la grande majorité de ces logements - les sept dixièmes - étaient les logements modestes ou très modestes hébergeant des familles à très bas revenu (National Low Income Housing Coalition, op. cit.). À leur place sont construits des logements de meilleure qualité, beaucoup plus chers.

Les personnes à la recherche de logements sociaux - public housing rencontrent encore plus de problèmes. Avant Katrina, 5100 familles vivaient dans des logements sociaux à La Nouvelle-Orléans (Bacon et Kornblum, 2006). En juin 2006, alors que la pression immobilière était très forte dans le sillage de Katrina, l'us Department of Housing and Urban Development et le HUD-controlled Housing Authority of New Orleans ont décidé paradoxalement de détruire les grands ensembles - public housing complexes - des quartiers de B. W. Cooper, C. J. Peete, Lafitte et St. Bernard, qui étaient en parfait état, pour les remplacer par des immeubles locatifs privés dont une partie seulement (moins de $50 \%$ ) seront des logements sociaux (Us Census Bureau cité par Warner, Krupa et Filosa, 2007). Il est vrai que ces opérations étaient déjà planifiées avant le passage du cyclone. Toutefois, leur mise en œuvre volontariste, sans tenir compte de la nouvelle situation, a été catastrophique pour les familles les plus pauvres.

\section{Des indemnités et des aides qui n'arrivent pas}

Un deuxième obstacle empêche le retour des IDPs qui le souhaitent : l'indemnisation des biens par les assurances est très inférieure aux pertes réelles subies. Souvent les assurances ne couvrent pas ou mal les dommages dus au vent ou aux inondations. Plus de 6600 contentieux ont été engagés auprès du tribunal fédéral de La Nouvelle-Orléans par des familles lésées. L'État de Louisiane a estimé que les propriétaires ont reçu en moyenne $4000 €$ de moins que ce qu'ils auraient dû obtenir, ce qui est énorme au regard de la faible valeur des biens en jeu (Eaton et Treaster, 2007). Dans son rapport The macroeconomic and budgetary effects of Hurricanes Katrina and Rita du 29 septembre 2005, le Congressional Budget Office (Commission des finances du Congrès) craignait que le paiement des assurances-inondations ne soit pas assez réactif. En réalité, $70 \%$ des demandes ont vite été traitées, 
mais pour des sommes largement insuffisantes ${ }^{3}$. Il convient de rajouter qu'une part non négligeable des populations pauvres n'était tout simplement pas assurée [http://www.urbanconservancy.org/library/pdf/katrina-twoyears-gulfblueprint.pdf]. Enfin, de nombreuses familles modestes habitant la même maison depuis des générations n'avaient plus de titres de propriété, voire n'en avaient jamais eu. Elles se sont donc retrouvées exclues des diverses aides.

Dans le même temps, les sommes promises n'arrivent pas. Au lendemain du cyclone, le gouvernement fédéral a accordé deux aides d'urgence pour un montant de 40 milliards d'euros et la FEMA une allocation plafonnée à $17000 €$ pour 60000 foyers sinistrés. Les financements fédéraux sont administrés directement par les États de Louisiane et du Mississippi, où les complications administratives se sont multipliées. La Louisiane a signé un contrat de 500 millions d'euros avec une société de Virginie - ICF International pour gérer ces fonds sous la dénomination the Road Home Program. Plus d'un an plus tard, aucune aide n'avait encore été versée. En février 2007 - 18 mois après Katrina - seules 630 personnes avaient reçu leur argent. En septembre 2007, moins de 50000 sur 150000 (Louisiana Road Home Program, 2007). Après quoi, le programme subit une coupe budgétaire de 2 milliards d'euros (Landrieu, 2007). De toute manière, dès décembre 2005, le fonds d'indemnisation public - National Flood Insurance Program - avait suspendu ses remboursements et la FEMA suspendait le paiement des 50000 chambres d'hôtel hébergeant les sinistrés. Plus de 10 mois après la catastrophe, le Congrès n'avait toujours pas ratifié une loi aidant les propriétaires à reconstruire leur logement.

La situation financière de nombreux IDPs est d'autant plus difficile que nombre d'entre eux n'ont pas retrouvé de travail. En août 2006, le taux de sans-emploi parmi eux était de $23 \%$. Comme le gouvernement fédéral a supprimé les allocations aux IDPs sans travail - plus de 64000 personnes -, la moitié des IDPs avaient en 2007 un revenu annuel inférieur à $10000 €$ (Louisiana Family Recovery Corps, op. cit.).

\section{Loin des yeux, loin du cour...}

Les IDPs qui parviennent à surmonter ces obstacles sont freinés par l'incertitude sur ce qui les attend au retour. Auront-ils à nouveau une épicerie, une école près de chez eux ? Retrouveront-ils leur communauté de voisinage ? Là non plus, les signaux ne sont pas rassurants.

3. Témoignage de David I. Maurstad, directeur et administrateur des assurances fédérales de la Mitigation Division (Federal Emergency Management Agency), devant le Committee on banking, housing, and urban affaires du Sénat des États-Unis, le 25 janvier 2006. 
Ainsi, les IDPs qui le souhaitent ont les plus grandes difficultés à participer à distance à la vie de leur communauté d'origine. Ils ne reçoivent aucune information sur les décisions concernant les programmes de reconstruction de logements, écoles, établissements de soins, aucune invitation aux réunions de quartier - les neighborhood planning meetings - (Kromm et Sturgis, 2007). Cet ostracisme s'est manifesté de la manière la plus brutale dans l'exercice du droit de vote, en avril 2006, lorsque La Nouvelle-Orléans a connu la première élection municipale après Katrina. À cette date, sur 299000 inscrits, près de 200000 étaient des IDPs (Louisiana Secretary of State, 2006). Des avocats des mouvements des droits civiques déposèrent une demande pour que des bureaux de vote soient créés dans les villes où était concentré un grand nombre IDPs (Houston, Atlanta, Memphis et Dallas). Le Département de la Justice refusa la requête puis, sous la pression de l'opinion, finit par autoriser 10 centres de vote, hors de La Nouvelle-Orléans mais tous dans l'État de Louisiane. De toute manière, ni les autorités fédérales ni celles de l'État n'envoyèrent de bulletins de vote ou d'informations sur les élections aux IDPs. Comme ces bureaux de vote ne disposaient pas de bulletins, il était impossible de voter à moins de faire une demande individuelle bien avant le scrutin (Clarke-Avery, 2006).

Le fait que les IDPs deviennent des migrants « sous contrainte » se traduit dans un taux impressionnant de troubles mentaux. Un échantillon de 815 IDPs a été examiné cinq mois puis un an et demi après la catastrophe par le Hurricane Katrina Community Advisory Group (CAG) dans le but d'examiner l'évolution du post-traumatic stress disorder (PTSD), les syndromes dépressifs et les tendances suicidaires (Kessler et al., 2008). Contrairement aux observations habituelles dans ce type d'évènement, le nombre et l'intensité des troubles, loin de diminuer avec le temps, persistent voire augmentent de manière significative.

\section{YOU'RE [NOT] WELCOME... UN ACCUEIL ENTRE SUSPICION ET REJET}

Le mal de vivre persistant des IDPs de Katrina peut s'expliquer par l'absence d'opportunités dans cet exil qui finit par devenir un état des choses. Mais là n'est pas la seule raison : ils ont été accueillis avec suspicion, peur, rejet voire violence.

\section{Préjugés et criminalisation dès les premières semaines}

Dans un premier temps, les évacués ont été regroupés dans des hébergements transitoires, les resettlement areas. Quelques jours après la catastrophe, alors que les évacués commençaient à peine à arriver, le gouverneur du Texas demanda au gouvernement fédéral le remboursement des coûts potentiels occasionnés. Puis, il demanda à la FEMA de ne plus leur envoyer 
d'IDPs au Texas (Contact the Press Office, 2005). Quant à la ville de Gretna, en Louisiane, proche voisine de La Nouvelle-Orléans, elle mit des policiers en position aux entrées de ville pour empêcher l'entrée des évacués (NPR, 2005). On peut facilement imaginer accueil plus chaleureux.

La vie dans les camps de transit n'était pas évidente. En 2006, l'ONG Save the Children a fait état de tensions extrêmes entre évacués et de graves crises familiales. Dans 5 sites sur 20, abus d'alcool, violences domestiques et abus sexuels présentaient des fréquences trois fois supérieures au pourcentage moyen observé dans les camps pour personnes déplacées similaires (Save the Children, 2006). Les camps étaient inhospitaliers (bleak and unwelcoming) : «Par exemple, Zirlott Park dans l'Alabama est surplombé de voies rapides et n'a aucune végétation. Les enfants n'ont pas d'aire de jeu ; on peut apercevoir un terrain de sport, mais l'accès en a été interdit par chaîne ${ }^{4}$. Seuls 3 sites sur 20 disposaient d'espaces publics et de salles de réunions. Enfin, la police pénétrait en permanence pour vérifier l'identité des personnes d'apparence Latino (Select Bipartisan Committee to Investigate the Preparation for and Response to Hurricane Katrina, 2005). De manière générale, une recherche systématique d'antécédents criminels était menée sur tous les IDPs. Cette procédure était légitimée par des médias populaires, comme en témoigne cet extrait de Fox News (2005) : "Dans tout le pays, les autorités des États et les autorités locales vérifient le passé des réfugiés (sic, ndt)... dans certains États (lesquels? ndt) la moitié des réfugiés ont un casier chargé $\gg^{5} \ldots$

Pourtant, cette suspicion relevait plus de préjugés que de réalités. Au Texas, l'État ayant accueilli le plus grand nombre d'IDPs, les autorités ont procédé à plus de 20000 contrôles. Très peu d'antécédents criminels ont été trouvés, toujours pour des délits mineurs. Les mêmes types de contrôles ont eu lieu dans le Massachusetts, le Tennessee et la Pennsylvanie avec les mêmes résultats. Cela n'a pas empêché les autorités des États de Rhode Island et de Caroline du Sud d'affirmer que plus de $50 \%$ des évacués avaient des antécédents criminels violents, alors que les contrôles effectués donnaient un chiffre beaucoup plus faible. Seule la Virginie-Occidentale a réellement trouvé $22 \%$ d'auteurs de crimes avec violence, mais sur un ensemble de seulement 350 évacués provenant d'un quartier de La Nouvelle-Orléans particulièrement dangereux. De nombreux États n'ont pas vérifié, du tout, les antécédents des IDPs, comme le Missouri, la Californie, le Maryland, le Minnesota et le Michigan.

4. «For example, Zirlott Park (Alabama) is dominated by broad roads and is without vegetation. The children have no playground but can see a ball field through a recently installed chain-link fence. »

5. "Around the nation, state and local authorities are checking refugees' pasts... In some states, half the refugees have rap sheets. » 


\section{Une hostilité généralisée}

Au-delà des discours compatissants, les relations des IDPs avec les populations locales ont donc été détestables d'entrée de jeu. Ces relations se sont encore dégradées lorsque les intérêts des uns et des autres s'opposaient, comme le montre le cas de Baton Rouge. La population de Greater BatonRouge s'est accrue de 200000 habitants dans la semaine qui a suivi le passage du cyclone (Social Security Administration, 2006). Plus de 100000 IDPs y sont restés. Une étude s'est intéressée à leur impact sur l'image que les résidents avaient de leur quartier et de leur ville, à partir des commentaires postés sur le plus fréquenté des sites américains de location (Russel N.J. III et Zahirovic-Herbert, 2008). Il apparait que l'arrivée des IDPs a été synchrone d'une chute brutale de satisfaction, à la fois sous la forme d'un indice global (la moyenne passant de 3 étoiles à 2 étoiles) et du pourcentage de résidents qui recommanderaient leur quartier à un ami (la moyenne passe de $50,4 \%$ à $27,4 \%)$.

Deux interprétations sont possibles. L'arrivée massive des IDPs a pu créer une tension du marché locatif qui s'est traduite par une plus forte occupation de logements de mauvaise qualité, qui n'auraient pas été loués autrement. D'ailleurs, les logements loués par les IDPs, dans l'urgence, étaient souvent d'une qualité inférieure à ceux qu'ils habitaient précédemment. Mais cette explication est contredite par le fait que la dégradation s'observe pour des logements identiques auparavant bien cotés, qui restent habités par leurs anciens résidents. La seconde interprétation est donc que l'insatisfaction est due à l'impression, pour les populations d'accueil, de ne plus « contrôler » leur environnement immédiat (Edwards et al., 1994). Le lien entre sentiment de contrôle de l'environnement et satisfaction des locataires a déjà été mis en évidence, dans un contexte plus général (LeBrasseur et al., 1988). La question du « contrôle » est particulièrement pertinente dans le cas de migrations résultant de catastrophes environnementales, tant pour les accueillants qui se sentent submergés, que pour les migrants qui ont perdu leurs repères.

La composition sociale et ethnique des IDPs de Katrina et la réputation de La Nouvelle-Orléans n'ont pas été sans amplifier le phénomène. La Nouvelle-Orléans c'était «the Big Easy », une cité de tolérance, «débauchée » pour une certaine Amérique puritaine. C'était également une ville où $68 \%$ de la population était afro-américaine. C'était, enfin, une ville où le revenu moyen par adulte était, pour certains quartiers, de moins de 8000 dollars par an, et où le taux d'homicides était le plus élevé de tous les États-Unis (10 fois supérieur à la moyenne nationale). 


\section{Houston, un cas d'école}

Le cas concret de la ville de Houston décrit bien comment une cohabitation tendue et conflictuelle a pu dégénérer en violences destructrices des liens sociaux. Houston, qui a accueilli le plus grand nombre d'IDPs, a connu un accroissement des crimes avec violence sans précédent en 2006 avec $28 \%$ d'homicides en plus. Le synchronisme avec l'arrivée des IDPs était troublant, puisque la tendance s'était amorcée dans les derniers mois de 2005. Immédiatement, la police incrimina les IDPs : «Lorsque les résidents de $\mathrm{La}$ Nouvelle-Orléans ont afflué à Houston, il y a six mois, pour échapper aux inondations, ils ont amené avec eux leurs gangs et la violence qui va avec ${ }^{6}$ (Kennett, 2006). Fortes de cette évidence, les autorités locales demandèrent des financements fédéraux pour renforcer la présence policière dans les quartiers où les IDPs étaient concentrés, tel Southwest Houston, qui regroupait $25 \%$ des 83300 IDPs, essentiellement dans des logements sociaux. Lorsque le gouvernement fédéral refusa la demande de Houston et que le sénateur Bond du Missouri demanda au Texas d'être «un bon voisin et non un gigolo $»^{7}$, le représentant Brady du Texas lui répondit : « Je ne me rappelle pas que le sénateur Bond ait ouvert le Saint Louis Dome aux victimes de Katrina ${ }^{8}$ (Associated Press, 2006). Cet échange de propos souligne l'atmosphère délétère autour du sort des déplacés de Katrina. Beaucoup d'habitants de Houston considèrent qu'ils ont nui à leur qualité de vie : $75 \%$ se disaient stressés et tendus, $67 \%$ accusaient les nouveaux venus de l'augmentation de la criminalité et un même nombre considérait que la situation allait empirer s'ils s'installaient définitivement (Houston Chronicle, 2006).

Pourtant, un examen même sommaire des faits révèle que, si l'augmentation de la criminalité est concomitante de l'arrivée des IDPs, le lien entre les deux évènements n'est ni évident ni direct. Sur 148 homicides, fin 2005, seulement 29 impliquaient des IDPs. De plus, parmi ces 29 cas, les IDPs étaient plus souvent victimes que suspects. Enfin, comme l'affirme Charles Rotramel, directeur de Youth Advocates, qui travaille avec des adolescents dans les banlieues à fort taux de criminalité : « Nous avions déjà des gangs et le niveau de violence avait déjà commencé à croître ${ }^{9}$. » En réalité, il semble que l'afflux brutal d'une population sinistrée a conduit les habitants de Houston à un sentiment de perte de contrôle sur leur environnement et à des conflits pour le partage d'un même espace de vie. Cela a engendré de la vio-

\footnotetext{
6. «When New Orleans residents streamed into Houston six months ago to escape the floodwaters caused by Hurricane Katrina, they brought in gangs and the violence that goes with them.»

7. «A good neighbor and not a paid companion.»

8. «I don't recall Senator Bond opening the St. Louis Dome to those Katrina victims. »

9. «We already had gangs, and the violence level was increasing already. »
} 
lence et une augmentation générale des comportements asociaux, extrêmes ou criminels. Dans cette affaire, les IDPs sont trois fois victimes : d'abord, par les préjugés qu'ils subissent ; ensuite, comme premières victimes de ces violences; enfin, comme boucs émissaires désignés, à tort, comme origine unique de cette vague criminelle.

Cette hostilité concerne la plupart des villes ayant reçu un grand nombre d'IDPs. Les solutions proposées en Californie ont contribué là où elles ont été appliquées à accroître malaise et violence : «Les résidents permanents sont en droit de percevoir les réfugiés (sic) comme des intrus et, après quelque temps, épuiser leur capital de sympathie à leur égard. Il est important de tout faire pour minimiser ce sentiment et cela est possible par deux manières. La première est de ségréger, cantonner, les réfugiés à des espaces bien limités, avec des forces de sécurité pour les protéger. L'autre est d'impliquer activement les communautés locales dans l'assistance et l'aide aux réfugiés » ${ }^{10}$ (Downey, 2007). C'est ainsi que, dans les écoles de Houston, les IDPs ont été placés dans des classes différentes (solution du cantonnement) et ont été invités à porter des bracelets spéciaux permettant de les signaler aux autres élèves, afin qu'ils les aident à s'intégrer (solution par l'implication des populations). Bien évidemment, le résultat a été catastrophique : le bracelet est devenu un signe infamant, les classes à part ont été perçues comme une mise en quarantaine, le fossé est devenu vite infranchissable entre les élèves IDPs et les autres. Les écoles de Houston ont connu en 2006 une douzaine d'altercations sérieuses, voire mortelles - est-il besoin de préciser que les IDPs étaient le plus souvent victimes -, et la présence policière dans les écoles a dû être augmentée de $10 \%$, selon l'Independent School District.

\section{CONCLUSIONS : PARTIR ENCORE...}

Le cyclone Katrina a été largement couvert par les médias américains, déclenchant une vague de sympathie et de pitié pour les victimes. Il est vrai que les catastrophes dites «naturelles », par la terminologie employée qui évoque un événement imparable et dramatique, non seulement dédouanent des responsabilités humaines et politiques dans l'exposition au risque, mais encore génèrent à court terme des sentiments consensuels de solidarité et de bienveillance envers les victimes. Pourtant, assez rapidement dans le cas des IDPs de Katrina, une franche hostilité s'est manifestée dans les espaces d'accueil.

10. «Permanent residents may view refugees as intruders and may, after a period of time, feel as though they have worn out their welcome. It is important to do everything possible to minimize that sentiment and this could be achieved in two possible ways. One would be to segregate the refugees in specified areas with some type of security for their protection. The other solution would be to involve the community in relief efforts to assist in providing for the basic needs of the refugees. » 
Le rejet dont ont fait l'objet les IDPs de Katrina de la part des communautés les accueillant trouve, certes, sa source dans le fait qu'ils sont majoritairement noirs, pauvres, venant de la ville la plus «débauchée » des États-Unis, par essence criminelle. De ce point de vue, ce qui est en jeu dans ce rejet, c'est la négation de celui qui cumule les stigmates (noir, pauvre, etc.).

Mais cela n'explique pas tout. Lors des catastrophes, les dispositifs de secours, d'assistance, d'évacuation ou de relogement se caractérisent, en général, par une propension à constituer les victimes en objets (Revet, 2008). Les multiples dysfonctionnements dans la gestion de cette crise due au cyclone Katrina ont rendu ce travers particulièrement visible et insupportable pour les victimes. Les protestations des dirigeants afro-américains des droits civiques sur l'usage du terme " réfugié » étaient, au fond, une manière de dire que les personnes touchées par Katrina avaient perdu leur statut de sujet. Ces protestations ont mis en lumière les responsabilités politiques et économiques dans l'occurrence et la forme de la crise. Dès lors, Katrina est devenu le révélateur de lignes de faille dans la société américaine et a généré une défiance à l'égard de ses institutions.

Dans le même temps, les IDPs ont commencé à produire leurs propres récits de l'événement et à revendiquer des droits spécifiques. Il convient de rappeler, en ce sens, la constitution dans l'après-Katrina d'une coalition d'organisations balayant un spectre assez large, depuis les mouvements des droits civiques aux associations de défense de l'environnement - le People's Hurricane Relief Fund -, revendiquant une implication politique des populations touchées dans la résolution des problèmes sociaux engendrés par Katrina [http ://www.peopleshurricane.org/].

Il est raisonnable de faire l'hypothèse qu'en quittant leur statut de victime, les IDPs ou les organisations qui s'en réclament ont construit une critique qui a donné à la société américaine - et particulièrement aux populations d'accueil - une mauvaise image d'elle-même, engendrant en retour une forte hostilité à l'égard des IDPs. Hostilité renforcée par un sentiment de perte de contrôle des populations d'accueil sur leur environnement immédiat, associé à des considérations économiques et à des rumeurs qui ont vite submergé la compassion initiale.

Or, une spécificité des IDPs de Katrina est que la plupart d'entre eux n'ont pas l'option du retour, face à la stratégie de reconstruction de La NouvelleOrléans qui gentrifie la ville et multiplie les obstacles à l'égard des IDPs - peu présentables - qui désireraient revenir. Importuns dans leur ville d'origine, malvenus dans leurs nouveaux lieux de résidence, les IDPs de Katrina ne peuvent s'installer durablement ni ici ni là. Leur seule alternative est de partir ailleurs, individuellement ou en famille, vers un endroit où ils ne seront plus visibles comme IDPs. C'est probablement la raison de leur redistribution, après coup. Il est remarquable que dans cette deuxième étape les personnes 
choisissent préférentiellement des villes et des États (Illinois, Caroline du Nord, Virginie, Alabama) à forte présence afro-américaine. On peut supposer que, dans leur choix, ils se sont appuyés sur leurs connaissances et leurs réseaux familiaux.

Ce faisant, si les IDPs de Katrina sont le produit d'une catastrophe environnementale et de facteurs politiques, économiques, sociologiques et psychologiques préexistants, les mécanismes en jeu ne sont pas bien différents de ceux à l'œuvre dans les migrations « classiques » (Weiner, 1992) :

- compétition entre natifs et migrants pour les logements, les emplois, l'espace, les ressources en général ;

- modification des équilibres entre les communautés déjà établies (s'il existe déjà un contentieux ou des préjugés, les tensions sont particulièrement fortes) ;

- soupçon réciproque des migrants pressentant un traitement discriminatoire à leur égard et des accueillants craignant un bouleversement de leur environnement.

Selon Richard Black et Khalid Koser (1998), des catastrophes environnementales sont cause ou conséquence de mouvements massifs de populations: cause, lorsque l'ancien espace de vie est devenu inhabitable; conséquence, lorsque les déplacements de population déclenchent une dégradation des conditions environnementales dans l'espace d'accueil. Le cyclone Katrina combine les deux aspects : en amont, la catastrophe et l'inondation de La Nouvelle-Orléans; en aval, la dégradation des conditions de vie - réelle ou fantasmée - dans les villes accueillant les IDPs avec surdensité et pression immobilière accrue. Il reviendra à un travail ultérieur mené dans le cadre du programme GICC (Gestion et impacts du changement climatique) du MEEDDAT - le projet EXCLIM (Exilés Climatiques) - de définir les parts respectives de ces facteurs et surtout comment ils s'articulent entre eux.

\section{RÉFÉRENCES BIBLIOGRAPHIQUES}

Associated PRess, 2006, March 12, «Appreciation Drying up for Texas Katrina Compassion ».

Bacon, J. ; Kornblum, J. 2006. «Plan To Raze Public Housing Sparks Suit », USA Today, June 28.

BECK, U. 2003. La Société du risque - Sur la voie d'une autre modernité, collection «Champs », Flammarion.

Black, R. ; Koser, K. 1998. Refugees, Environment and Development, Addison Wesley, New York.

BRoder, J.M. 2005. «Amid Criticism of Federal Efforts, Charges of Racism are Lodged », The New York Times, September 5.

CAdoul, T. ; MARET, I. 2008. «Résilience et reconstruction durable: que nous apprend La Nouvelle-Orléans?», Les Annales de Géographie, $\mathrm{n}^{\circ}$ 663, p. 104-124. 
Clarke-Avery, K. 2006. "Rebuilding and the Ballot: Race and Politics after Katrina », dans C. Kromm (sous la dir. de), One Year after Katrina: The State of New Orleans and the Gulf Coast, p. 24-25, Institute for Southern Studies, Durham, North Carolina.

Conisbee, M. ; Simms, A. 2003. Environmental Refugees. The Case for Recognition, New Economics Foundation, London.

Contact the Press Office. 2005. «Summary of Key State of Texas Actions in Response to Hurricane Katrina », September 3, Texas Governor Press Release, Austin.

Downey, M.T. 2007. Is the Prospect of Urban Refugees in America Real? Can Rural Law Enforcement Meet the Challenge? Command College Independent Study Project, California Commission on Peace Officer Standards and Training.

EATON, L. ; Treaster, J.B. 2007. «Insurers Bear Brunt Of Anger in New Orleans », The New York Times, September 3.

Edwards, J. ; Fuller, T. ; Sermsi, S. ; VorakitPhoKatorn, S. 1994. « Why People Feel Crowded: An Examination of Objective and Subjective Crowding », Population and Environment, vol. 16, $\mathrm{n}^{\circ} 2$, p. 149-173, Springer.

El-Hinnawi, E. 1985, Environmental Refugees, United Nations Publications, New York.

Fox News. 2005, September 22. « Half Katrina Refugees Have Records ».

Houston Chronicle. 2006, March 24. « Houstonians Evacuee-Weary, Poll Says ».

Kennet, J. 2006. «Louisiana Gangs That Fled Katrina Heighten Houston Murder Rate », Bloomberg, March 3. http://www.bloomberg.com/apps/news?pid= $10000103 \&$ sid=az6n8C6gsqf0\&refer $=$ us

Kessler, R.C. ; Galea, S. ; Gruber, M.J. ; Sampson, N.A. ; Ursano, R.J. ; Wessely, S. 2008. «Trends in Mental Illness and Suicidality after Hurricane Katrina », Molecular Psychiatry, n 13, p. 374-384, Nature Publishing Group.

Kromm, C. ; Sturgis, S. 2007. Blueprint for Gulf Renewal: The Katrina Crisis and a Community Agenda for Action, Institute for Southern Studies, Durham, North Carolina.

LANDRIEU, M. (Us Senator) 2007. «Our \$ 12 Billion Day », Press Release from US Senate, November 8.

LeBrasseur, R. ; Blackford, K. ; Whissell, C. 1988. « The Leford Test of Tenant Locus of Control: Introducing an Effective Measure Relating Locus of Control and Housing Satisfaction », Environment and Behavior, vol. 20, n 3, p. 300319, Sage.

Lipton, E. 2005. « FEMA Calls 60000 Houses In Storm Area Beyond Repair », The New York Times, November 5.

Louisiana FAmily Recovery CorPs. 2007, August 2007. Where Did They Go and Are They Coming Back? An Analysis of Displaced Louisianans Living in Other States, Baton Rouge. http://www.recoverycorps.org/media/files/WhereDidThey Go.pdf

Louisiana Road Home Program. 2007. Program Statistics As Of February 12, 2007, Baton Rouge.

Louisiana SeCRETARY OF STATE. 2006, May 22. «Weekly voter registration statistics », Press Release, Baton Rouge. 
MAnCEBo, F. 2006. « Katrina et La Nouvelle-Orléans : entre risque "naturel” et aménagement par l'absurde », Cybergeo, $\mathrm{n}^{\circ} 353$, rubrique « Géographie appliquée et aménagement" », Paris. http://193.55.107.45/articles/353res.htm

MCGREGOR, J. 1995. « Are There Environmental Refugees? », Refugee Participation Network, $\mathrm{n}^{\circ} 18$, p. 7-10, Oxford University, Oxford.

McQuAID, J. 2006. « Katrina's Assault on New Orleans », World Watch, n 19, p. 1317, World Watch Institute, Washington DC.

Meitrodt, J. 2006. «Rising Rents », New Orleans Times-Picayune, October 15.

National Low Income Housing Coalition. 2005. Hurricane Katrina's Impact on Low Income Housing Units, Research Note \#05-02.

NPR. 2005, September 20. «Evacuees Were Turned Away at Gretna, La », http://www.npr.org/templates/story/story.php?storyId=4855611

REVET, S. 2008. «La vulnérabilité, une notion problématique. Un regard d'anthropologue », colloque interdisciplinaire Vulnérabilités, Toulouse, 14, 15 et 16 mai.

RusSEL, N.J. III ; ZAHIROVIC-HERBERT, V. 2008. « The Impact of a Migration Shock on Tenant Satisfaction: The Case of Hurricane Katrina and Baton Rouge, Louisiana », Migration Letters, vol. 5, $\mathrm{n}^{\circ}$ 1, p. 79-88, London.

Save the Children. 2006. Rapid Assessments of Temporary Housing Camps For Hurricane-Displaced Children and Families, July 2006.

http://www.savethechildren.org/publications/reports/katrina-assessment _final-0706.pdf

Select Bipartisan Committee to Investigate the Preparation for and Response to Hurricane Katrina. 2005. «Written Testimony by Bill Chandler of the Mississippi Immigrants Rights Alliance and Guadalupe Gamboa of Oxfam American », Dec. 6.

Social Security Administration. 2006. The Social Security Administration's Service Delivery to Individuals and Beneficiaries Affected by Hurricanes Katrina and Rita: Evaluation report (A-06-06-26072), Office of the Inspector General Washington DC.

Suhrke, A. ; Visentin, A. 1991. «The Environmental Refugee: A New Approach », Ecodecision, $\mathrm{n}^{\circ}$ 2, p. 73-84, Montréal.

SwaIN, A. 1996. "Environmental Migration and Conflict Dynamics: Focus on Developing Regions », Third World Quarterly, n 17, p. 959-973, Routledge, London.

The Brookings Institution and Greater New Orleans Community Data Center. 2007. The New Orleans Index: Tracking Recovery in the Region, New Orleans. http://www.brookings.edu/metro/pubs/200512_katrinaindex.htm

us Census Bureau. 2006. Special Population Estimates for Impacted Counties in the Gulf Coast Area, Hurricane Data and Emergency Preparedness, Washington DC, dans C. Warner; M. Krupa et G. Filosa, 2007. «Demolition Protests Ignore Some Realities », New Orleans Times-Picayune, December 15.

WeINER, M. 1992. « Security, Stability, and International Migration », International Security, $\mathrm{n}^{\circ}$ 17, p. 91-126, Belfer Center, Harvard University, Cambridge.

White House. 2006. The Federal Response to Hurricane Katrina: The Lessons Learned, February 23, Assistant to the President for Homeland Security and Counter-terrorism, Washington DC. 